\title{
CONSISTENCY TEST AND CONSTRAINT OF QUINTESSENCE
}

\author{
CHIEN-WEN CHEN \\ Department of Physics, National Taiwan University \\ Taipei 10617, Taiwan, R.O.C \\ f90222025@ntu.edu.tw \\ JE-AN GU \\ Leung Center for Cosmology and Particle Astrophysics (LeCosPA), \\ National Taiwan University, Taipei 10617, Taiwan, R.O.C. \\ PISIN CHEN \\ Kavli Institute for Particle Astrophysics and Cosmology, \\ SLAC National Accelerator Laboratory, Menlo Park, CA 94025, U.S.A. and \\ Department of Physics, Graduate Institute of Astrophysics and LeCosPA, \\ National Taiwan University, Taipei 10617, Taiwan, R.O.C.
}

\begin{abstract}
In this paper we highlight our recent work in arXiv:0803.4504. In that work, we proposed a new consistency test of quintessence models for dark energy. Our test gave a simple and direct signature if certain category of quintessence models was not consistent with the observational data. For a category that passed the test, we further constrained its characteristic parameter. Specifically, we found that the exponential potential was ruled out at the $95 \%$ confidence level and the power-law potential was ruled out at the $68 \%$ confidence level based on the current observational data. We also found that the confidence interval of the index of the power-law potential was between -2 and 0 at the $95 \%$ confidence level.
\end{abstract}

Keywords: Consistency test; quintessence; dark energy.

PACS No.: 95.36.+x

\section{Introduction}

Compelling evidences from Type Ia supernovae (SNe Ia) and other cosmological observations show that the expansion of the universe is undergoing an accelerating stage at late times (see Ref. 1 for reviews). Within the framework of general relativity, assuming inhomogeneity and isotropy, this indicates that there should exist an energy source, termed dark energy, which provides a significant negative pressure to cause this acceleration. Thus far the nature of dark energy remains unresolved and is generally regarded as one of the most tantalizing problems in cosmology. While a positive cosmological constant remains the simplest realization of dark energy, current observational data have not ruled out the possibility of a time-evolving dark energy. $^{2}$ 
Quintessence, a time-varying scalar field,$^{3-5}$ is one of the leading alternatives for dark energy. Its energy density and equation of state generally evolve with time. There are various categories of quintessence with different potential forms (for example, see Ref. 6-10) that have been proposed. Studies of classification, ${ }^{11,12}$ and the general dynamical behavior ${ }^{13}$ of quintessence have been carried out. There have been also works on the reconstruction of quintessence potentials ${ }^{14-18}$ and the investigations of how future observational data can better constrain individual categories of quintessence. ${ }^{8-10}$ While these works have helped us understand the nature of quintessence and obtain constraints of the potential form, a method to assess whether and how different categories of quintessence are consistent with the observational data should be beneficial.

Recently we proposed a new method to test individual categories of quintessence. ${ }^{18}$ For each category of quintessence, we suggested to introduce a characteristic $Q(z)$ which in general can vary in redshift but is equivalent to a constant parameter within the domain of the category. We further defined the measure of consistency, $\mathcal{M}(z)$, as the derivative of $Q(z)$ with respect to the redshift $z$. The observational data should allow a null value for $\mathcal{M}(z)$ if the corresponding category of quintessence is consistent with them. If, however, the $\mathcal{M}(z)=0$ line lies outside certain confidence region of the data, that category of quintessence is ruled out by the observational data at the corresponding confidence level.

To demonstrate this method, in Ref. 18 we performed the test on the exponential potential (see Ref. 10 and the references therein) and the power-law potential. ${ }^{6,7}$ To obtain the constraint on the measure of consistency $\mathcal{M}(z)$ from the data, a parametrization of dark energy was required. In Ref. 18 we invoked a broadly used form of the parameterization of the equation of state $w(z),{ }^{19,20}$

$$
w(z)=w_{0}+w_{a}(1-a)=w_{0}+w_{a} z /(1+z) .
$$

For a particular category of quintessence that passes the test, we can further constrain its characteristic parameter by reconstructing $Q(z)$. We found that this method could effectively rule out some of the categories of quintessence. The process and the results are shown in the next section.

\section{Consistency Test and Constraint of Quintessence}

Quintessence field and the potential are related to the equation of state as follows. ${ }^{18,21}$

$$
\begin{gathered}
\phi(z)-\phi_{0}= \pm \int_{0}^{z} \frac{\sqrt{\left[1+w\left(z^{\prime}\right)\right] \rho_{\phi}\left(z^{\prime}\right)}}{H\left(z^{\prime}\right)} \frac{d z^{\prime}}{1+z^{\prime}} \\
V(z)=[1-w(z)] \rho_{\phi}(z) / 2
\end{gathered}
$$

where $\rho_{\phi}(z)$ is the energy density of the quintessence field and $H(\mathrm{z})$ is the Hubble expansion rate. 
We performed the consistency test of the exponential potential and the powerlaw potential, respectively,

$$
\begin{aligned}
V_{\exp }(\phi) & =V_{A} \exp [-\phi / M], \\
V_{\text {power }}(\phi) & =m^{4-n} \phi^{n} .
\end{aligned}
$$

For the exponential potential, we identified $M$ in Eq. (4) as the characteristic parameter and accordingly defined the characteristic $Q_{\exp }(z)$, which in general would evolve with the redshift but is equivalent to the constant parameter $M$ within the exponential potential paradigm,

$$
\begin{aligned}
Q_{\exp }(z) & \equiv-V(z)\left(\frac{d V}{d \phi}\right)^{-1}(z) \\
& =M \quad \text { for the exponential potential. }
\end{aligned}
$$

We then defined the measure of consistency as the derivative of the this characteristic quantity with respect to the redshift,

$$
\begin{aligned}
\mathcal{M}_{\exp }(z) & \equiv \frac{d Q_{\exp }}{d z}(z) \\
& =0 \text { for the exponential potential. }
\end{aligned}
$$

For the consistency test of the exponential potential, we reconstructed $\mathcal{M}_{\exp }(z)$ via the constraints of $w_{0}$ and $w_{a}$ deduced from the observational data, with the use of the equations above. If $\mathcal{M}_{\exp }(z)=0$ lies outside certain confidence region, the exponential potential is not consistent with the observation at the corresponding confidence level.

In the same spirit, we identified the following characteristic quantity corresponding to the index of the power-law potential,

$$
\begin{aligned}
Q_{\text {power }}(z) & \equiv\left[1-V(z)\left(\frac{d V}{d \phi}(z)\right)^{-2} \frac{d^{2} V}{d \phi^{2}}(z)\right]^{-1} \\
& =n \text { for the power-law potential. }
\end{aligned}
$$

The measure of consistency of the power-law potential was then defined as

$$
\begin{aligned}
\mathcal{M}_{\text {power }}(z) & \equiv \frac{d Q_{\text {power }}}{d z}(z) \\
& =0 \quad \text { for the power-law potential. }
\end{aligned}
$$

In Ref. 18, we adopted the constraints of $w_{0}$ and $w_{a}$ obtained by Riess et al. ${ }^{22}$ We found that the consistency measure of the exponential potential, $\mathcal{M}_{\exp }(z)$, lay outside the $95 \%$ confidence region while that of the power-law potential, $\mathcal{M}_{\text {power }}(z)$, lay outside the $68 \%$ but inside the $95 \%$ confidence regions (see Ref. 18 for details). We further constrained the index $n$ of the power-law potential by reconstructing $Q_{\text {power }}(z)$ using Eqs. (10) and (11). The value of $n$ was found to be between -2 and 0 at the $95 \%$ confidence level. 
Following the work in Ref. 18, recently we invoked the constraints of $w_{0}$ and $w_{a}$ that we obtained by analyzing the up-to-date combined data sets from SNe Ia, ${ }^{22-24}$ cosmic microwave background, ${ }^{25}$ and baryon acoustic oscillation. ${ }^{26}$ Under these new constraints, the exponential potential was still ruled out at the $95 \%$ confidence level. The result is shown in Fig. 1 (see Ref. 27 for details).

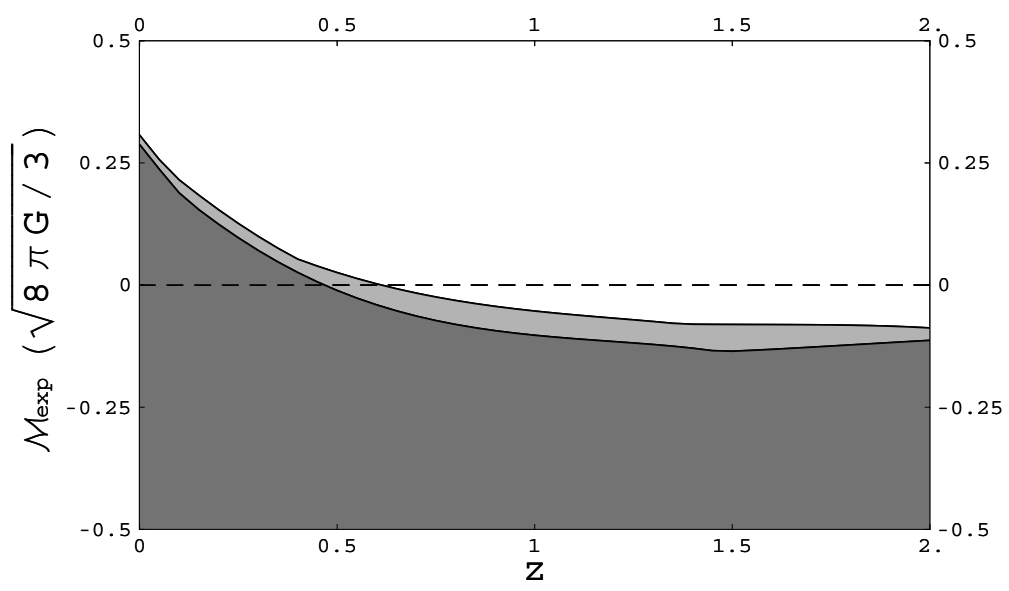

Fig. 1. The consistency measure of the exponential potential. The dark gray area corresponds to the $68 \%$ confidence region while the light gray corresponds to the $95 \%$ confidence region. The null line of the measure lies outside the $95 \%$ confidence region. This indicates that the exponential potential is ruled out at the $95 \%$ confidence level.

\section{Summary}

In Ref. 18 we proposed a new consistency test of quintessence by introducing a consistency measure $\mathcal{M}(z)$ for each category of the quintessence models. It was efficiently done via the constraint of a single set of parameters from the observational data without solving the equation of motion of quintessence. If a specific category of quintessence passes the test, we can further constrain its characteristic parameter by reconstructing the characteristic quantity $Q(z)$ without reliance on the knowledge of other parameters of the category and the initial conditions.

It was shown that the exponential potential was ruled out at the $95 \%$ confidence level and the power-law potential was ruled out at the $68 \%$ confidence level based on the current observational data. We further found that the $95 \%$ confidence interval of the power index of the power-law potential was between -2 and 0 . Future observations may give tighter constraint.

This method of consistency test can be applied not only to other categories of quintessence but also to other categories of dark energy models. In Ref. 27, we apply this method on five categories of dark energy models, including cosmological 
constant, three categories of quintessence, and the generalized Chaplygin gas. The discriminating power and the possible bias of this method among different categories of dark energy models can also be studied via the Monte Carlo test. ${ }^{27}$

\section{References}

1. J. Frieman, M. Turner and D. Huterer, arXiv:0803.0982 [astro-ph].

2. A. Albrecht et al., arXiv:astro-ph/0609591.

3. R. R. Caldwell, R. Dave and P. J. Steinhardt, Phys. Rev. Lett. 80, 1582 (1998) [arXiv:astro-ph/9708069].

4. Je-An Gu and W-Y. P. Hwang, Phys. Lett. B 517, 1 (2001) [arXiv:astro-ph/0105099].

5. L. A. Boyle, R. R. Caldwell and M. Kamionkowski, Phys. Lett. B 545, 17 (2002) [arXiv:astro-ph/0105318].

6. I. Zlatev, L. Wang and P. J. Steinhardt, Phys. Rev. Lett. 82, 896 (1999) [arXiv:astro$\mathrm{ph} / 9807002]$.

7. P. J. Steinhardt, L. Wang and I. Zlatev, Phys. Rev. D 59, 123504 (1999) [arXiv:astro$\mathrm{ph} / 9812313]$.

8. M. Barnard, A. Abrahamse, A. Albrecht, B. Bozek and M. Yashar, Phys. Rev. D 77, 103502 (2008) [arXiv:0712.2875 [astro-ph]].

9. A. Abrahamse, A. Albrecht, M. Barnard and B. Bozek, Phys. Rev. D 77, 103503 (2008) [arXiv:0712.2879 [astro-ph]].

10. B. Bozek, A. Abrahamse, A. Albrecht and M. Barnard, Phys. Rev. D 77, 103504 (2008) [arXiv:0712.2884 [astro-ph]].

11. R. R. Caldwell and E. V. Linder, Phys. Rev. Lett. 95, 141301 (2005) [arXiv:astro$\mathrm{ph} / 0505494]$.

12. E. V. Linder, Phys. Rev. D 73, 063010 (2006) [arXiv:astro-ph/0601052].

13. D. Huterer and H. V. Peiris, Phys. Rev. D 75, 083503 (2007) [arXiv:astro-ph/0610427].

14. M. Sahlen, A. R. Liddle and D. Parkinson, Phys. Rev. D 75, 023502 (2007) [arXiv:astro-ph/0610812].

15. D. Huterer and M. S. Turner, Phys. Rev. D 60, 081301 (1999) [arXiv:astro$\mathrm{ph} / 9808133]$.

16. T. D. Saini, S. Raychaudhury, V. Sahni and A. A. Starobinsky, Phys. Rev. Lett. 85, 1162 (2000) [arXiv:astro-ph/9910231].

17. B. F. Gerke and G. Efstathiou, Mon. Not. Roy. Astron. Soc. 335, 33 (2002) [arXiv:astro-ph/0201336].

18. Je-An Gu, C.-W. Chen and P. Chen, arXiv:0803.4504 [astro-ph].

19. E. V. Linder, Phys. Rev. Lett. 90, 091301 (2003) [arXiv:astro-ph/0208512].

20. M. Chevallier and D. Polarski, Int. J. Mod. Phys. D 10, 213 (2001) [arXiv:grqc/0009008].

21. Z. K. Guo, N. Ohta and Y. Z. Zhang, Phys. Rev. D 72, 023504 (2005) [arXiv:astro$\mathrm{ph} / 0505253]$.

22. A. G. Riess et al., Astrophys. J. 659, 98 (2007) [arXiv:astro-ph/0611572].

23. W. M. Wood-Vasey et al. [ESSENCE Collaboration], Astrophys. J. 666, 694 (2007) [arXiv:astro-ph/0701041].

24. T. M. Davis et al., Astrophys. J. 666, 716 (2007) [arXiv:astro-ph/0701510].

25. E. Komatsu et al. [WMAP Collaboration], arXiv:0803.0547 [astro-ph].

26. D. J. Eisenstein et al. [SDSS Collaboration], Astrophys. J. 633, 560 (2005) [arXiv:astro-ph/0501171].

27. C.-W. Chen, Je-An Gu and P. Chen (in preparation). 